\title{
Anaesthetic method and short-term outcomes of preterm infants delivered by caesarean section at a tertiary hospital in South Africa
}

\author{
R Stander, MB ChB, DCH (SA); L Tooke, MB ChB, FCPaed (SA), MMed (Paed), Cert Neonatol (SA); \\ A R Horn, MB ChB, DCH (SA), FCPaed (SA), Cert Neonatol (SA), PhD \\ Division of Neonatal Medicine, School of Child and Adolescent Health, Faculty of Health Sciences, University of Cape Town, South Africa
}

Corresponding author: A R Horn (alan.horn@uct.ac.za)

\begin{abstract}
Background. There are inconsistent published data describing the influence of maternal anaesthetic type during caesarean section (CS) on outcomes of preterm neonates.

Objectives. To describe indications and type of anaesthesia in preterm neonates, and their short-term outcomes, comparing spinal anaesthesia (SA) to general anaesthesia (GA).

Methods. Data were collected retrospectively on preterm babies born at 28 - 35 weeks' gestation by CS between 1 January and 30 September 2014 at Groote Schuur Hospital, Cape Town, South Africa. Babies with missing data were excluded. The largest group of babies with similar indications for delivery were identified from the theatre register. Baseline characteristics and short-term outcomes for this group were extracted from an existing prospective data base, and compared between those delivered under SA and GA.

Results. Data were available for 226 deliveries, having excluded 23 with incomplete data. Most babies (75\%) were delivered under SA. The most common indication for CS was 'cardiotocograph abnormalities', in 139 deliveries. Within this group, SA was the more frequent $(81.7 \%$ v. $12.9 \%)$, while GA was associated with lower Apgar scores $(p<0.001)$ and more intubation at birth $(p=0.004)$. There was no difference in mortality when comparing SA with GA.

Conclusion. Our data suggest a sedative effect of maternal GA on preterm babies delivered by CS, and the need for staff with advanced resuscitation skills. This study provides novel baseline data in our setting, but these data need to be validated in a prospective study.
\end{abstract}

S Afr J Child Health 2019;13(2):125-129. https://doi.org/10.7196/SAJCH.2019.v13i3.1606

Prematurity is a leading cause of morbidity and mortality globally, and particularly in developing countries. ${ }^{[1]}$ Caesarean section (CS) of preterm neonates implies increased risk due to the indications for operative delivery, such as intrapartum emergencies, fetal hypoxia, maternal illness and maternal medication. The influence of the type of maternal anaesthesia on outcomes in this group may assist with the appropriate assignment of medical staff, but current data are conflicting. ${ }^{[2,3]}$

The effect of general anaesthesia (GA) and regional anaesthesia (RA) on short-term outcomes of neonates after CS was studied in a Cochrane review that was updated in 2012. ${ }^{[2]}$ Regional anaesthesia included both epidural and spinal anaesthesia (SA). The authors found no difference in 1-minute and 5-minute Apgar scores, but most of the included studies either compared outcomes in term babies only, or they did not analyse data from preterm babies separately. A more recent study in Nigeria found that GA was an independent risk factor for low Apgar scores in neonates of $<36$ weeks' gestation. ${ }^{[3]}$ A low Apgar score indicates the need for skilled medical personnel to be present at the delivery, ${ }^{[4,5]}$ and is strongly associated in both preterm and term neonates with neonatal and infant death, when compared with other predictable variables. ${ }^{[6-8]}$

At Groote Schuur Hospital (GSH), a tertiary hospital in South Africa, both GA and are performed for preterm caesarean delivery. We designed a descriptive study to obtain baseline data in our setting, comparing SA to GA. The objectives of this study were to: (i) to describe the indications for CS and the type of anaesthesia in a cohort of preterm neonates; (ii) to identify a subgroup of babies defined by a common indication for CS; and (iii) to describe the need for resuscitation and related short-term outcomes in the uniform subgroup, comparing SA with GA.

\section{Methods}

\section{Study design and ethics approval}

A retrospective study was conducted on a cohort of preterm CS deliveries from 1 January to 30 September 2014. The study was a descriptive one, to generate novel baseline data in our setting. A 9-month period of study was identified as being the most accessible and recent period with complete data at the time of data collection. In addition, this period was expected to generate a sample of $\sim 200$ neonates.

The study was approved by the University of Cape Town Health Sciences Faculty Human Research Ethics Committee (ref. no. 598/2015), and conforms to the principles of the 2013 Declaration of Helsinki. ${ }^{[9]}$

\section{Study setting}

GSH is a tertiary academic hospital in Cape Town, South Africa. Obstetric services include a dedicated obstetric theatre for both elective and emergency CSs with 24-hour coverage. Neonatal services include a 75-bed neonatal unit with intensive care facilities and 200 admissions per month. At GSH, the type of anaesthesia for CS (SA or GA) is decided by the attending anaesthesiologist and may be determined by the clinical and physical condition of mother and/or fetus. The anaesthetic is usually performed by an anaesthetic registrar, supported by an on-site consultant or senior registrar. Neonatal resuscitation is provided by an intern or medical officer, supported by an on-site registrar. A senior medical officer or registrar is expected to attend if intrapartum hypoxia or a birth weight of $<1200 \mathrm{~g}$ is expected.

\section{Population}

All preterm babies born at 28 - 35 weeks' gestational age with a birth weight of $<1500 \mathrm{~g}$ and delivered by CS under GA or SA (not epidural 
anaesthesia) were included. Neonates with missing data regarding indication for CS or method of anaesthesia were excluded.

\section{Data collection and analysis}

The type of anaesthesia utilised and the indications for CS were obtained from the obstetric theatre register. The largest subgroup of neonates with similar indications for delivery were identified; baseline characteristics and outcome data for this subgroup were extracted from a prospectively maintained data base of very low birth weight neonates $(<1500 \mathrm{~g})$ admitted in GSH. The database forms part of the Vermont Oxford Network Database (VON). Neonatal units from around the world submit data to the VON, through clinical review of medical records according to common definitions, to facilitate anonymous data comparison. ${ }^{[10]}$

The main indication for CS was determined - where more than one indication was listed, that based on preserving fetal well-being was noted as the primary one. Mothers with cardiotocograph (CTG) abnormalities described as 'fetal distress', 'pathological CTG' and 'non-reassuring CTG' were grouped in a single group named 'CTG abnormalities'. All data were derived from the theatre register and the VON database. CTGs were not available for review. When sentinel events (acute fetal emergencies) such as abruptio placentae, cord prolapse and fetal bradycardia were present, these were listed as the primary indication.

Neonatal baseline characteristics included birth weight, gestational age and gender. Maternal characteristics included antenatal care, treatment with antenatal steroids, treatment with magnesium sulphate, maternal hypertension, gestational proteinuric hypertension $(\mathrm{GPH})$ and multiple gestation. The neonatal outcomes included 1-minute and 5-minute Apgar scores, oxygen administration during resuscitation, intubation in the delivery room, cardiac compression in the delivery room, surfactant administration, oxygen administration on day 28 , severe periventricular haemorrhage, cystic periventricular leukomalacia, necrotising enterocolitis, pneumothorax and mortality.

The data from the theatre register were captured into an Excel (Microsoft, USA) spreadsheet and matched to data extracted from the GSH portion of the VON using folder numbers.

Stata version 12 (StataCorp, USA) was used for statistical analysis. Indications for CS were described using frequency distributions and compared according to mode of anaesthesia. A relatively uniform subgroup of neonates with similar indications for CS, in which maternal and neonatal characteristics, and short-term perinatal outcomes, were matched, were compared by mode of anaesthesia. The relationship between maternal anaesthetic type and neonatal outcomes was compared. Categorical variables were compared using the Fisher's exact test or $\chi^{2}$, depending on the expected values. Continuous variables were compared using Student's $t$-test for parametric variables and the Wilcoxon Rank Sum test for non-parametric variables, respectively. All tests were two-sided with significance set at $p<0.05$.

\section{Results}

There were 249 preterm CS deliveries during the 9-month study period. Data describing the method of anaesthesia and/or the indication for CS were incomplete or missing for 23 deliveries. Of the remaining 226 CS deliveries, $24.8 \%(n=56)$ were delivered under GA and $75.2 \%(n=170)$ under SA.

The indications for CS are shown in Table 1. The most common indication for CS was 'CTG abnormalities', irrespective of the method of anaesthesia. GA was used relatively more frequently when acute fetal emergencies such as abruptio placentae or cord prolapse were present.

The 139 CS deliveries performed due to 'CTG abnormalities' formed the largest, most relatively uniform subgroup for which we could most reasonably compare outcomes of SA with GA. The majority of deliveries in this subgroup (87.1\%) were done under SA. GA was used significantly more frequently in deliveries due to abruptio placentae, GPH, intrauterine growth restriction and placenta praevia. There was a trend towards increased frequency of GA in deliveries due to cord prolapse and extra-uterine pregnancy. The baseline characteristics of the 'CTG abnormalities' subgroup are shown in Table 2 . The only significant difference was that antenatal magnesium sulphate was used more frequently in the neonates delivered by GA. There was a trend towards a marginally higher gestational age at birth in the neonates delivered by SA.

Resuscitation requirements, Apgar scores and other short-term outcomes of the 'CTG abnormalities' group are shown in Table 3. Both the 1-minute and the 5-minute Apgar scores were significantly lower in the GA group. Oxygen administration and intubation during resuscitation also both occurred significantly more frequently in the GA group. There was a trend towards increased oxygen requirement on day 28 in the GA group, but there were no significant differences in mortality or any of the other outcome variables.

Table 1. Indication for CS in preterm delivery

\begin{tabular}{|c|c|c|c|}
\hline Primary indications & Total deliveries $(N=226), n(\%)$ & GA $(n=56), n(\%)$ & SA $(n=170), n(\%)$ \\
\hline CTG abnormalities* & $139(61.5)$ & $18(32.1)$ & $121(71.2)$ \\
\hline Abruptio placentae & $7(3.1)$ & $5(8.9)$ & $2(1.2)$ \\
\hline Cord prolapse & $2(0.9)$ & $2(3.6)$ & $0(0)$ \\
\hline Fetal bradycardia $(<100 \mathrm{bpm})$ & $2(0.9)$ & $1(1.8)$ & $1(0.6)$ \\
\hline Previous CS & $8(3.5)$ & $2(3.6)$ & $6(3.5)$ \\
\hline GPH & $26(11.5)$ & $13(23.2)$ & $13(7.6)$ \\
\hline IUGR & $3(1.3)$ & $3(5.4)$ & $0(0)$ \\
\hline Abnormal lie & $16(7.1)$ & $2(3.6)$ & $14(8.2)$ \\
\hline $\mathrm{APH}$ & $2(0.9)$ & $0(0)$ & $2(1.2)$ \\
\hline Maternal cardiac/pulmonary oedema & $1(0.4)$ & $1(1.8)$ & $0(0)$ \\
\hline Extra-uterine preg. & $2(0.9)$ & $2(3.6)$ & $0(0)$ \\
\hline Multiple preg. & $9(4.0)$ & $2(3.6)$ & $7(4.1)$ \\
\hline Placenta previa & $7(3.1)$ & $7(12.5)$ & $0(0)$ \\
\hline Failed IOL & $2(0.9)$ & $1(1.8)$ & $1(0.6)$ \\
\hline
\end{tabular}


Table 2. Baseline characteristics in the cohort with CTG abnormalities $(N=139)$

\begin{tabular}{|c|c|c|c|}
\hline Characteristics & GA $(n=18), n(\%)^{*}$ & SA $(n=121), n(\%)^{*}$ & $p$-value \\
\hline \multicolumn{4}{|l|}{ CTG description } \\
\hline Fetal distress & $17(94.4)$ & $95(78.5)$ & 0.197 \\
\hline Pathological & $1(5.6)$ & $14(11.6)$ & 0.692 \\
\hline Non-reassuring & $0(0)$ & $12(9.9)$ & 0.365 \\
\hline \multicolumn{4}{|l|}{ Neonatal characteristics } \\
\hline Birth weight (grams), median (IQR) & $1215(1000-1310)$ & $1200(1040-1360)$ & 0.880 \\
\hline Gestational age (weeks), median (IQR) & $30(28-32)$ & $31(30-32)$ & 0.056 \\
\hline Male sex & $8(44.4)$ & $58(47.9)$ & 0.782 \\
\hline \multicolumn{4}{|l|}{ Maternal characteristics } \\
\hline Antenatal care & $15(83.3)$ & $106(87.6)$ & 0.705 \\
\hline Antenatal steroids & $17(94.4)$ & $109(90.1)$ & 1.000 \\
\hline Antenatal $\mathrm{MgSO}_{4}$ & $13(72.2)$ & $45(37.2)$ & 0.009 \\
\hline Maternal HPT & $15(83.3)$ & $85(70.2)$ & 0.399 \\
\hline Maternal GPH & $6(33.3)$ & $24(19.8)$ & 0.222 \\
\hline
\end{tabular}

A box plot showing the distribution of Apgar scores at 1 minute in the GA and SA groups is shown in Fig. 1. The box plot shows that $25 \%$ of the neonates in the GA group had a 1-minute Apgar of 1, which was also the lowest recorded Apgar.

\section{Discussion}

The most frequent primary indication for preterm caesarean delivery in the entire cohort was 'CTG abnormalities'. Spinal anaesthesia was used almost three times more frequently than GA in the entire cohort, and more than five times more frequently in the subgroup of neonates delivered due to 'CTG abnormalities'. The neonates in this subgroup who were delivered using SA had higher 1- and 5-minute Apgar scores and required less resuscitation in the delivery room than those delivered using GA. Our findings are in keeping with global trends, including countries with similar health economics there is a shift towards the use of SA for CS, with the aim of avoiding both maternal and fetal complications. ${ }^{[3,11]}$

Mothers with acute sentinel events, such as abruptio placenta and cord prolapse, were almost exclusively delivered by GA - this may have been because some experts consider GA to be the preferred method in fetal and maternal emergency scenarios owing to a fast and reliable induction. ${ }^{[12]}$ The potential poorer outcomes in these neonates could be expected to skew the outcomes in the GA towards poorer, but these neonates were not included in the 'CTG abnormalities' group. The rationale for the preference for GA when performing CS for fetal emergencies was challenged in the retrospective observational study of preterm neonates in Nigeria by Nwafor et al. ${ }^{[3]}$ - in this study, preterm neonates delivered by emergency CS under GA had significantly lower Apgar scores than those where SA was used.

There are significant challenges in interpreting Apgar scores. Population-based cohort studies have determined that $95.2 \%$ of preterm neonates have a 5 -minute Apgar score of $\geq 7,{ }^{[6]}$ and $98.8 \%$ of neonates have a 5-minute Apgar score of $\geq 7$ after normal vertex delivery. ${ }^{[8]}$ The potential association between a low 5-minute Apgar score, fetal hypoxia and mortality ${ }^{[8]}$ makes it difficult to ascertain whether a low 5-minute Apgar score is a consequence of the GA or the underlying fetal state. Most of the data comparing mode of anaesthesia and neonatal outcomes includes the term neonates, but Apgar scores in preterm neonates are usually lower than term neonates and so should be considered separately. ${ }^{[2,13]}$

A large Australian study had a similar approach and similar findings to those of our study. Algert et al. ${ }^{[7]}$ showed that GA was

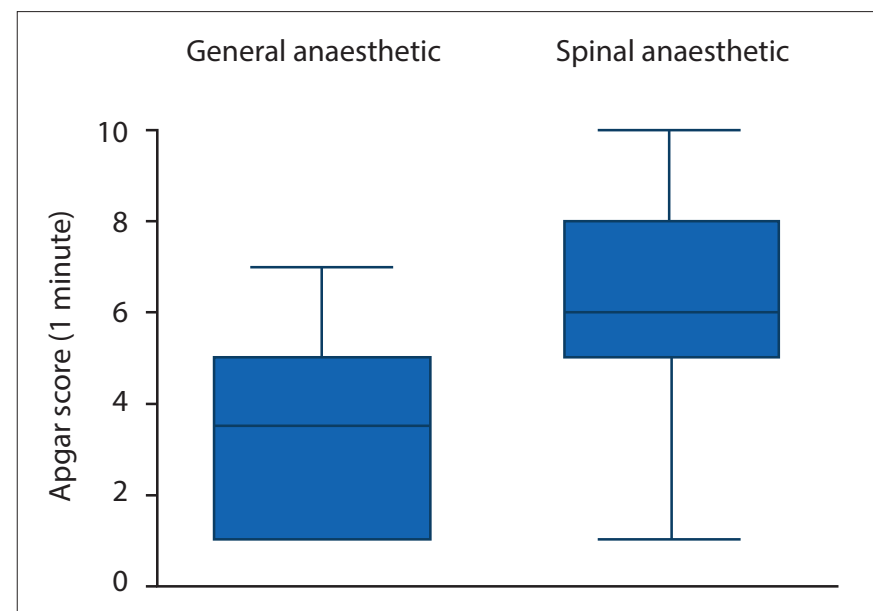

Fig. 1. One-minute Apgar scores after general and spinal anaesthetic.

associated with increased frequency of intubation and lower Apgar scores, in both term and preterm neonates. They similarly controlled for confounding factors by specifying pregnancy risk and indications for CS, and they found that the neonatal benefits from the use of RA (spinal and epidural anaesthesia were analysed as single group) for CS persisted across a range of delivery indications, i.e. planned repeat CS, failure to progress and fetal distress. The authors suggest a sedative anaesthetic effect. ${ }^{[7]}$

The level of experience and skill of practitioners attending newborns after CS delivery varies depending on the delivery circumstances and the available resources, but the attendance of an experienced midwife is the norm for uncomplicated vaginal deliveries. ${ }^{[14]}$ A prospective South African study at GSH in 2010 found that complicated deliveries that included multiple pregnancy, low birthweight, prematurity, general anaesthetic, abnormal lie or known congenital abnormality had a higher resuscitation rate $(45 \%) \cdot{ }^{[14]}$ Similarly, an observational study in 2005 found that after both emergency and elective CS, significantly more infants required resuscitation when GA was used. ${ }^{[15]}$ Our findings concur with these studies, which recommend that skilled practitioners are present for all CSs under GA because of the association with low Apgar score and need for resuscitation. ${ }^{[14,15]}$

The Cochrane database analyses showed a similar proportion of neonates with Apgar scores $<6$, in both term and preterm neonates by 
Table 3. Short term outcomes in the cohort with CTG abnormalities $(N=139)$

\begin{tabular}{|c|c|c|c|}
\hline Outcomes & GA $(n=18), n(\%)^{*}$ & SA $(n=121), n(\%)^{*}$ & $p$-value \\
\hline One-minute Apgar, median (IQR) & $3.5(1-5)$ & $6(5-8)$ & $<0.001$ \\
\hline Five-minute Apgar, median (IQR) & $7(6-8)$ & $9(8-10)$ & $<0.001$ \\
\hline Oxygen during resuscitation & $18(100)$ & $93(76.9)$ & 0.024 \\
\hline ETT delivery room & $5(27.8)$ & $5(4.1)$ & 0.004 \\
\hline Adrenaline delivery room & $0(0)$ & $1(0.8)$ & 1.000 \\
\hline CPR delivery room & $3(16.8)$ & $10(8.3)$ & 0.376 \\
\hline Surfactant any time & $7(38.9)$ & $30(24.8)$ & 0.253 \\
\hline Oxygen on D28 ( $n=56)$ & $4(22.2)$ & $6(5)$ & 0.080 \\
\hline Pneumothorax $(n=149)$ & $0(0)$ & $2(1.7)$ & 1.000 \\
\hline $\operatorname{NEC}(n=149)$ & $2(11.1)$ & $7(5.8)$ & 0.329 \\
\hline Severe IVH $(n / N=13 / 110)$ & $0(0)$ & $7(7)$ & 1.000 \\
\hline Cystic PVL ( $n / N=13 / 113)$ & $0(0)$ & $3(3)$ & 1.000 \\
\hline Deaths & $2(11.1)$ & $11(9.1)$ & 0.676 \\
\hline
\end{tabular}

CS when comparing GA with RA. However, the indications for delivery varied from uncomplicated pregnancies delivered electively at term to emergency CSs, so the data interpretation is limited by heterogeneity. ${ }^{[2]}$

We considered the possibility that the significantly higher use of antenatal magnesium sulphate in the GA subgroup in our study may have confounded the difference in the Apgar scores. Magnesium sulphate is currently recommended for use in women at risk of imminent preterm birth, as it has neuroprotective properties for the fetus in this setting and is associated with a reduced risk of cerebral palsy ${ }^{[16]}$ The beneficial effect of magnesium sulphate might therefore be thought to be associated with higher Apgar scores, but high fetal magnesium levels might also be expected to cause respiratory depression at birth. A systematic review and meta-analysis of five studies published in 2017 found no difference in the frequency of low Apgar scores at 5 minutes, and no difference in the need for resuscitation at birth. ${ }^{[17]}$

With respect to mortality, we found no difference between the two anaesthetic methods - in keeping with findings from an observational study in a Nigerian teaching hospital, ${ }^{[3]}$ but in contrast to a large population-based cohort study of very preterm neonates (the EPIPAGE cohort), where mortality was increased in the SA group. ${ }^{[13]}$ The authors postulated that associated hypotension and placental hypoperfusion could account for poorer outcomes, and that term neonates may tolerate placental hypoperfusion better. ${ }^{[13]} \mathrm{In}$ our study, the strong association between GA and decreased Apgar scores, in the face of no significant difference in deaths, suggests the presence of a sedative effect of GA on the neonates.

The labour ward register did not have data on uterine incision to delivery time, so we are not able to comment on a potential effect that may have had on neonatal outcomes. However, a prospective 2-year cohort study of 812 in-labour CSs of non-anomalous term neonates did not find an association between incision to delivery time and hypoxia-associated morbidities. ${ }^{[18]} \mathrm{A}$ secondary analysis of a prospective cohort of 793 CSs, including both term and preterm neonates, compared outcomes of neonates who had an incision to delivery interval of $\geq 2$ minutes with those with shorter intervals, and found that gestational age was the only variable independently associated with neonatal morbidity ${ }^{[19]}$

\section{Study strengths and limitations}

Our study has several limitations. It is a retrospective study with some missing data and the potential for selection bias. The theatre register did not include data regarding pre- or intra-operative maternal complications that might have affected Apgar scores, but the primary indication for delivery was neonatal in all cases. Although maternal hypotension can be more frequent in SA, this group had better outcomes, so this is unlikely to have played a role. The study is observational and not powered for a specific outcome. The 'CTG abnormalities' group may not be entirely homogenous, but the criteria for inclusion in this group were defined, and babies with acute fetal emergencies were excluded. The generalisability of the findings is limited both to the setting and the population - this is a single hospital-based study at a tertiary referral centre with a high-risk population of mothers and very low birth-weight preterm neonates.

The study also has significant strengths; firstly, it identified a group of neonates with relatively uniform indications for operative delivery; secondly, the exclusion of term neonates further enhanced homogeneity; and thirdly, important maternal and neonatal variables that could affect outcomes were included.

\section{Conclusion}

CTG abnormalities constituted the most frequent indication for CS delivery of preterm neonates, and SA was the most frequently used method of anaesthesia. GA was strongly associated with lower Apgar scores compared with SA, and this study provides novel baseline data in our setting. Our data suggest a sedative effect of maternal GA on preterm babies, but prospective, matched, case-control studies are needed to provide more robust results. Our data could be used to inform the design and power of such studies. Irrespective of the cause of the low Apgar scores, our data suggest that staff with advanced resuscitation skills should be present at all preterm deliveries where GA is used.

Declaration. This manuscript was submitted as a requirement for an MMed degree.

Acknowledgements. Thank you to the GSH theatre staff for facilitating access to records.

Author contributions. RS collected the data, did some of the data analysis and wrote the paper. All authors had input to the protocol design and critically reviewed the manuscript. ARH assisted with data analysis and interpretation. LT maintains and provided data from the VON database. 
Funding. Partly funded by University of Cape Town.

Conflicts of interest. None.

1. World Health Organization. Atlas of African Health Statistics 2016 - health situation analysis of the African Region. http://www.aho.afro.who.int/en/ publication/5266/atlas-african-health-statistics-2016-health-situationanalysis-african-region (accessed 25 March 2017).

2. Afolabi BB, Lesi FEA. Regionalv. general anaesthesia for caesarean section. Cochrane Database Syst Rev 2012;(10):CD004350. https://doi.org/10.1002\%2F14651858. cd004350.pub3

3. Nwafor MI, Aniebue UU, Nwankwo TO, Onyeka TC, Okafor VU. Perinatal outcome of preterm caesarean section in a resource-limited centre: A comparison between general anaesthesia and subarachnoid block. Niger J Clin Pract 2014;17(5):613-618. https://doi.org/10.4103/1119-3077.141428.

4. Rolbin SH, Cohen MM, Levinton CM, Kelly EN, Farine D. The premature infant. Anesth Analg 1994;78(5):912-917. https://doi.org/10.1213\% 2F00000539-199405000-00013.

5. Tudjegbe SO, Imarengiaye CO, Sadoh, WE. Determinants of paediatrician's presence at caesarean section. West Afr J Med 2012;31(1):24-27.

6. Casey BM, McIntire DD, Leveno KJ. The continuing value of the Apgar score for the assessment of newborn infants. Obstet Gynecol Surv 2001;56(7):406407. https://doi.org/10.1097\%2F00006254-200107000-00010.

7. Algert CS, Bowen JR, Warwick GB, Knoblanche GE, Lain SJ, Roberts CL. Regional block versus general anaesthesia for caesarean section and neonatal outcomes: A population-based study. BMC Med 2009;7(1):20. https://doi org/10.1186\%2F1741-7015-7-20.

8. Iliodromiti S, Mackay DF, Smith GCS, Pell JP, Nelson SM. Apgar score and the risk of cause-specific infant mortality: A population-based cohort study. Lancet 2014;384(9956):1749-1755. https://doi.org/10.1016\%2 Fs0140-6736\%2814\%2961135-1.

9. World Medical Association. World Medical Association Declaration of Helsinki. Ethical Principles for Medical Research Involving Human Subjects. JAMA 2013;310(20):2191-2194. https://doi.org/10.1001/ jama.2013.281053
10. Horbar J, Soll R, Edwards WH. The Vermont Oxford Network: A community of practice. Clin Perinatol 2010;37(1):29-47. https://doi.org/10.1016\%2Fj. clp.2010.01.003

11. Imarengiaye C, Asudo F, Akinmola A, Lawal, B. A snap-shot survey of spinal anaesthesia for caesarean section: The Nigeria experience. J Clin Sci 2017;14(4):173-177. https://doi.org/10.4103\%2Fjcls.jcls_46_17

12. Saygi Aİ, Özdamar Ö, Gün İ, Emirkadi H, Müngen E, Akpak YK. Comparison of maternal and fetal outcomes among patients undergoing cesarean section under general and spinal anesthesia: A randomized clinical trial. Sao Paulo Med J 2015;133(3):227-234. https://doi.org/10.1590\%2F1516-3180.2014.8901012

13. Laudenbach V, Mercier FJ, Rozé JC, et al. Anaesthesia mode for caesarean section and mortality in very preterm infants: An epidemiologic study in the EPIPAGE cohort. Int J Obstet Anesth 2009;1(2):142-149. https://doi.org/10.1016\%2Fj. ijoa.2008.11.005

14. Tooke LJ, Joolay Y, Horn AR, Harrison MC. Is the attendance of paediatricians at all elective caesarean sections an effective use of resources? S Afr Med J 2011;101(10):749-750

15. Gordon A, McKechnie EJ, Jeffrey H. Pediatric presence at cesarean section: Justified or not? AJOG 2005;193(3):599-605. https://doi.org/10.1016\%2Fj. ajog.2005.06.013

16. Doyle LW, Crowther CA, Middleton P, Marret S, Rouse D. Magnesium sulphate for women at risk of preterm birth for neuroprotection of the fetus. Cochrane Database Syst Rev 2009;1:CD004661. https://doi.org/10.1002\%2F14651858. cd004661.pub3

17. Crowther CA, Middleton PF, Voysey M, et al. Assessing the neuroprotective benefits for babies of antenatal magnesium sulphate: An individual participant data meta-analysis. PLoS Med 2017;14(10):e1002398. https://doi. org/10.1371\%2Fjournal.pmed.1002398

18. Spain J, Tuuli M, Stout M, et al. Time from uterine incision to delivery and hypoxic neonatal outcomes. Am J Perinatol 2015;32(05):497-502. https:// doi. org/10.1055/s-0034-1396696

19. DiNapoli M, Moroz L, Son M, D’Alton M, Gyamfi-Bannerman C. Poster Session V. AJOG 2015;212(S):S403. https://doi.org/10.1016/j.ajog.2014.10.1046

Accepted 9 January 2019. 